\title{
Mice lacking the syndecan-3 gene are resistant to diet-induced obesity
}

\author{
April D. Strader, ${ }^{1}$ Ofer Reizes, ${ }^{2}$ Stephen C. Woods, ${ }^{1}$ Stephen C. Benoit, ${ }^{1}$ and Randy J. Seeley ${ }^{1}$ \\ 'Department of Psychiatry, University of Cincinnati, Cincinnati, Ohio, USA. ${ }^{2}$ Procter \& Gamble Pharmaceuticals, Mason, Ohio, USA.
}

\begin{abstract}
The accurate matching of caloric intake to caloric expenditure involves a complex system of peripheral signals and numerous CNS neurotransmitter systems. Syndecans are a family of membrane-bound heparan sulfate proteoglycans that modulate ligand-receptor interactions. Syndecan-3 is heavily expressed in several areas of the brain, including hypothalamic nuclei, which are known to regulate energy balance. In particular, syndecans have been implicated in modulation of the activity of the melanocortin system, which potently regulates energy intake, energy expenditure, and peripheral glucose metabolism. Our data demonstrate that syndecan-3-null mice have reduced adipose content compared with wild-type mice. On a high-fat diet, syndecan-3-null male and female mice exhibited a partial resistance to obesity due to reduced food intake in males and increased energy expenditure in females relative to that of wild-type mice. As a result, syndecan3-null mice on a high-fat diet accumulated less adipose mass and showed improved glucose tolerance compared with wild-type controls. The data implicate syndecan-3 in the regulation of body weight and suggest that inhibition of syndecan-3 may provide a therapeutic approach for the treatment of obesity resulting from exposure to high-fat diets.
\end{abstract}

\section{Introduction}

Body adipose mass is regulated by matching caloric intake to caloric expenditure over time. The CNS is a critical site where signals of adipose stores are sensed and where appropriate changes in intake and/or expenditure are produced. Over the past decade, a number of proteins have been implicated in the process of sensing peripheral fuel status and the effector mechanisms that defend peripheral adipose mass (1). While most of these proteins are neurotransmitters, receptors, or intracellular signaling molecules, recent data indicate an important role for a unique family of proteins called syndecans. Syndecans are ubiquitous cell surface heparan sulfate proteoglycans (HSPGs; proteins with covalently attached, highly acidic sugar chains), unique in their ability to bind many extracellular peptides, such as hormones and growth factors. Syndecans are found on almost every cell type (2) but are differentially expressed depending on the tissue type.

Evidence for the involvement of syndecans in the control of energy balance comes from mice that overexpress syndecan- 1 (3). Despite using the pan-selective cytomegalovirus promoter enhancer, Reizes and colleagues showed that mice overexpressing syndecan- 1 express transgenic syndecan-1 in a highly unique and circumscribed pattern including expression in regions of the hypothalamus that have long been linked to energy balance regulation, such as the paraventricular nucleus $(3,4)$. Quite unexpectedly, these mice also show a profound, maturity-onset obesity and type 2 diabetes (3). Although such data indicate a potential role for syndecans in the CNS control of energy balance, syndecan-1 is not normally expressed in the CNS. However, other members of the syndecan family are expressed in the CNS and, most nota-

Nonstandard abbreviations used: AgRP, Agouti-related protein; HF, high-fat; HSPG, heparan sulfate proteoglycan; IPGTT, intraperitoneal glucose tolerance test; LF, low-fat; MC, melanocortin.

Conflict of interest: The authors have declared that no conflict of interest exists.

Citation for this article: J. Clin. Invest. 114:1354-1360 (2004).

doi:10.1172/JCI200420631. bly, syndecan-3 can be found in some of the same hypothalamic regions in which syndecan- 1 appeared in the transgenic mice. Furthermore, syndecan-3 is highly homologous $(5,6)$ to the cytoplasmic and transmembrane domains of syndecan-1, and hypothalamic expression of syndecan-3 is increased by fasting (3). Given these findings, we hypothesized that the profound obesity of mice overexpressing syndecan-1 exposes a role for syndecan-3 in the CNS circuitry that regulates energy balance. To test this hypothesis, we sought to determine the phenotype of mice with targeted genetic disruption of syndecan-3.

When humans or rodents are exposed to highly palatable and calorically dense high-fat (HF) diets, the amount of stored fat in the body increases (7-9). Because such diets probably contribute to the rising levels of obesity in the developed world, identifying the proteins involved in the maintenance of increased adipose stores and impaired glucose tolerance that occurs when animals are exposed to HF diets has become an important focus of obesity research. Thus, a crucial question about syndecan-3 is whether it is involved in the deleterious effects of HF diets on both maintenance of increased adipose stores and glucose tolerance. To that end, we compared syndecan-3-deficient (syndecan-3-/-) mice and their wild-type controls on both low-fat (LF) and HF diets for their food intake, body weight, energy expenditure, body composition, and glucose tolerance.

\section{Results}

Resistance to diet-induced obesity in syndecan-3-/- mice. HF diets lead to increased adipose stores in rodents and humans. Therefore, we studied the effects of diet on energy homeostasis in syndecan-3null mice. Seven-week-old male and female wild-type (C57BL/6J) and syndecan- $3^{-1-}$ (congenic with the C57BL/6J strain) mice were given unlimited access to one of two diets (LF, $11 \%$ fat by calories, and HF, $41 \%$ fat by calories) for a period of 14 weeks. Weekly body weight measurements revealed that syndecan-3-/- mice appeared to be relatively resistant to the weight gain caused by HF diet exposure observed in the wild-type mice (Figure 1). Wild-type mice on 

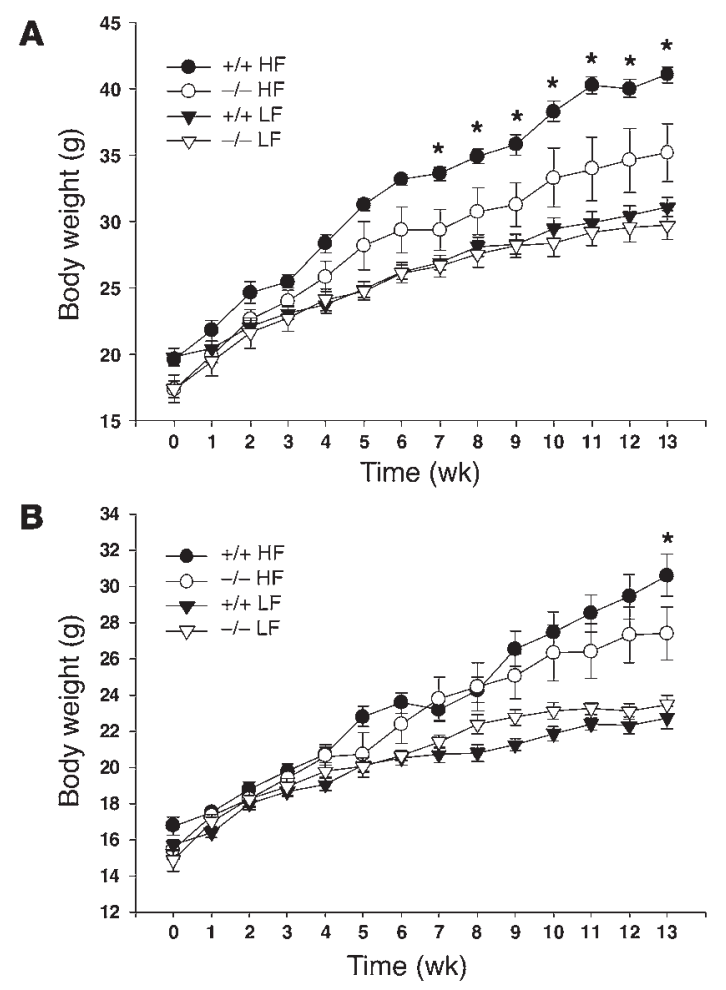

the HF diet had increased body weight typical of that observed when animals are placed on this dietary regimen. Syndecan-3deficient mice also gained weight on the HF diet; however, the amount gained was significantly less (Figure 2) than that gained by wild-type mice at 14 weeks. Interestingly, the difference in body weight gain between wild-type and syndecan-3-1- mice appeared to be greater in males than females. In our study, carcass analysis was performed on a subset of animals from the 14-week diet study (HF groups only) and showed that the increased body mass seen in wild-type mice after long-term exposure to the HF diet was due mainly to an increase in body fat and a small increase in lean body mass (Figure 2 and Table 1). Female syndecan-3-/- mice and wildtype mice had similar amounts of lean body mass at the end of 14 weeks on the HF diet. However, male wild-type mice had greater lean body mass than did syndecan-3-1- mice (Table 1 ).

Interestingly, mice heterozygous for syndecan-3 were also partially protected from the effects of the HF diet. Syndecan-3 heterozygotes were bred to yield wild-type, syndecan-3+-, and syndecan-3-1- mice. In this second study, female mice of each genotype were placed on the HF diet for 10 weeks. Body weight and body composition were measured at weekly intervals with a rodent NMR body composition analyzer. By 10 weeks on the HF diet, female homozygous (syndecan-3---) and heterozygous (syndecan-3+/-) mice had a significantly lower percent body fat than that of wildtype mice (Figure 3 ). These data corroborate the initial findings and demonstrate that haploinsufficiency at the syndecan-3 locus appears to partially protect mice from weight gain on a HF diet, similar to complete syndecan-3 deficiency.

syndecan-3-/- mice have less body fat on the LF diet. Interestingly, the body weights approached but did not reach significance by 10 weeks in the second study involving heterozygous females, yet the syndecan-3 $3^{+-}$and syndecan-3 $3^{-/-}$mice still had lower body fat percentages than those of wild-type mice (Table 1). Given the finding

\section{Figure 1}

Body weight gain of syndecan-3-/- and wild-type mice on LF (11\% fat) and HF (41\% fat) diets. (A and B) Male (A) and female (B) syndecan-3-/- $(-/-)$ and wild-type $(+/+)$ mice $(n=6-10$ in each group) were given ad libitum access to diets for 14 weeks $\left({ }^{*} P<0.05\right.$, repeated-measures two-way ANOVA; gene-diet-sex interaction, $-\mathrm{df}=(1$, 50); $\mathrm{F}=7.72 ; P=0.007)$.

that body composition was different despite similar body weights, we then examined the body compositions of mice raised on rodent chow (also low in fat; $15 \%$ fat by calories). Body composition was determined in male and female syndecan-3-1- and wild-type mice that were 12 weeks of age and also 28 weeks of age (male only). Surprisingly, syndecan-3-deficient mice were significantly leaner and had less body fat than wild-type mice, even on a diet low in fat. At 12 weeks of age, female syndecan-3-deficient mice had significantly less body fat and greater lean mass than wild-type mice (Figure 4 and Table 1); however, this difference was not significant in male mice. By 28 weeks of age, male syndecan- $3^{-/-}$mice had considerably less body fat than age-matched wild-type mice (Figure 4 and Table 1).

Feeding behavior of syndecan-3-/- mice. Given that the mice on either the LF or HF diet were not housed individually, we were unable to accurately measure individual daily food intake in this cohort of animals. In a separate cohort of adult age- and weight-matched wild-type and syndecan-3-1- mice, we assessed whether the reason for the resistance of syndecan-3-/- mice to the development of dietinduced obesity was reduced calorie intake. Figure 5 demonstrates that wild-type and syndecan-3-/- mice of both sexes consumed the same kcal of LF diet. In contrast, male wild-type mice consumed a greater amount of HF diet than did male syndecan-3-/- mice. Surprisingly, female wild-type and syndecan- $3^{-/-}$mice consumed the same amount of HF diet.

Energy expenditure of syndecan-3-1- mice. Another potential explanation for the resistance of syndecan-3 $3^{-/}$mice to the development of diet-induced obesity is an increase in metabolic rate. We performed indirect calorimetry to determine whether syndecan- $3^{-/-}$mice have different metabolic rates than those of wild-type mice. Adult mice maintained on a HF diet for 4 weeks were placed in the indirect calorimeter for 24 hours with access to ad libitum food (HF diet) and water. Both male and female syndecan-3-1- mice exhibited increased energy expenditure (kcal) compared with that of wild-type mice

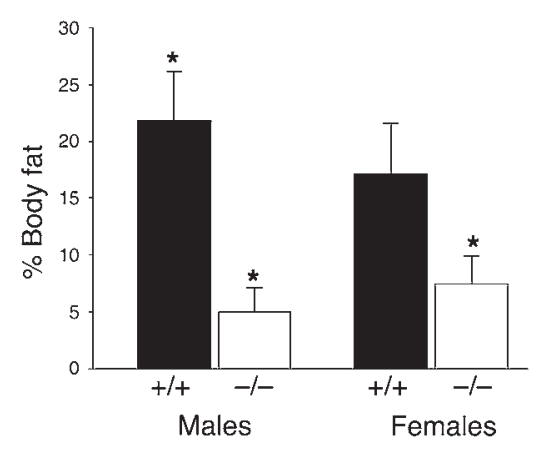

Figure 2

Percent body fat of syndecan-3-1- $(n=3)$ and wild-type $(n=3)$ mice after 14 weeks on the HF diet ( ${ }^{\star} P<0.05$ for both females and males, Student's $t$ test). 
Table 1

Body composition of mice following LF and HF diets

\begin{tabular}{|c|c|c|c|c|c|}
\hline Sex & Genotype & Diet & Final body wt (g) & Lean $(\mathrm{g})^{\mathrm{A}}$ & Fat (g) \\
\hline \multirow[t]{2}{*}{ Male $^{B}$} & $+/+$ & HF & $37.12 \pm 1.27$ & $11.2 \pm 1.4^{\mathrm{C}}$ & $6.3 \pm 1.7$ \\
\hline & $-1-$ & HF & $28.16 \pm 0.88$ & $8.0 \pm 0.3$ & $1.4 \pm 0.5^{c}$ \\
\hline \multirow[t]{2}{*}{ Female $^{B}$} & $+/+$ & HF & $27.04 \pm 2.84$ & $7.1 \pm 0.3$ & $6.1 \pm 1.8$ \\
\hline & $-1-$ & HF & $21.71 \pm 0.34$ & $6.1 \pm 0.1$ & $2.1 \pm 0.6^{C}$ \\
\hline \multirow[t]{3}{*}{ Female } & $+/+$ & HF & $24.28 \pm 0.46$ & $18.5 \pm 0.5$ & $4.3 \pm 0.5$ \\
\hline & $+/-$ & $\mathrm{HF}$ & $23.03 \pm 0.73$ & $18.5 \pm 0.3$ & $2.9 \pm 0.5$ \\
\hline & $-1-$ & $\mathrm{HF}$ & $21.58 \pm 1.53$ & $17.7 \pm 1.0$ & $2.6 \pm 0.4^{c}$ \\
\hline Male & $+/+$ & Chow & $29.88 \pm 0.58$ & $25.6 \pm 0.8$ & $1.1 \pm 0.4$ \\
\hline (12 wk) & $-1-$ & Chow & $31.37 \pm 0.92$ & $27.4 \pm 1.1$ & $1.1 \pm 0.2$ \\
\hline Female & $+/+$ & Chow & $19.18 \pm 0.20$ & $16.6 \pm 0.2$ & $0.9 \pm 0.2$ \\
\hline (12 wk) & $-1-$ & Chow & $19.76 \pm 0.30$ & $17.5 \pm 0.2^{\mathrm{C}}$ & $0.5 \pm 0.1^{c}$ \\
\hline Male & $+/+$ & Chow & $33.82 \pm 0.70$ & $26.0 \pm 0.4$ & $5.3 \pm 0.7$ \\
\hline (7 mo) & $-1-$ & Chow & $30.05 \pm 0.67$ & $25.9 \pm 0.6$ & $1.2 \pm 0.3^{C}$ \\
\hline
\end{tabular}

ATwo separate methods to measure body composition were used. BMale and female mice $(-/-)$ and $(+/+)$ on the HF diet were analyzed using the ether extraction method. Water weight was determined separately after lyophilization of the carcass; therefore, lean weight or non-fat weight is lower compared to the other groups. All other groups were analyzed using the rodent NMR. In these groups, lean weight includes water weight. ${ }^{C} P<0.05$ compared to the other groups of mice in each study.

much weight as did the wild-type controls (Figures 1 and 3). The difference in body weight was mainly due to an increase in percent body fat in wild-type mice (Figures 2 and 4). Thus, on the HF diet, male syndecan-3-1- mice had $75 \%$ less body fat and female syndecan-3 $3^{-/}$mice had $50 \%$ less body fat than wildtype mice (Figure 2 and Table 1). Notably, even in the absence of an obvious body weight difference, as seen with the female mice fed the HF diet for 10 weeks (Figure 3) and the regular chow-fed mice (Figure 4), syndecan-3-/- mice still had reduced body fat stores compared with those of wild-type mice (Figure 4 and Table 1). Haploinsufficiency also appeared to partially prevent female mice from gaining excess body fat stores on the HF diet (Figure 3 and Table 1).

While syndecan-3 $3^{-/}$mice showed resistance to obesity on the HF diet, males and females relied on different mechanisms. Male syndecan-3-/- mice consumed significantly less calories of HF diet than wild-type mice consumed, yet the numbers of calories of the HF diet consumed by female syndecan-3-/- mice were comparable to those consumed by their wild-type controls (Figure 5). In contrast, while male syndecan-3--mice showed a significant increase in energy expen-

during both the light and dark cycles (Figure 6). Notably, the magnitude of the increase in metabolism was substantially greater for females than for males. This is consistent with the observation that the food intake of female wild-type and syndecan-3-/- mice was comparable. Thus, the difference in body fat for the females must have been due to a larger difference in energy expenditure.

Effect of HF diet on glucose tolerance. Some of the physiological consequences of diet-induced obesity are insulin resistance, glucose intolerance, and the eventual development of overt diabetes. We evaluated whether syndecan-3-1- mice showed any changes in glucose tolerance after prolonged exposure to the HF diet. To test this, we performed an intraperitoneal glucose tolerance test (IPGTT) between weeks 14 and 16 on a cohort of mice raised on the HF diet. Wild-type and syndecan-3-1- mice were made to fast overnight (16-19 hours) and received an intraperitoneal injection of $1.5 \mathrm{~g} / \mathrm{kg}$ body weight $20 \%$ D-glucose. Tail blood was collected for measurement of plasma glucose and insulin. Consistent with their having reduced adipose stores, male and female syndecan-3-/mice fed the HF diet had improved glucose clearance after the glucose challenge (Figure 7) compared with the glucose clearance of wild-type controls. Additionally, fasting plasma glucose and insulin were measured at the time of sacrifice. Despite similar plasma glucose levels, syndecan-3-deficient mice had significantly lower plasma insulin compared to that of wild-type mice (Figure 8). Fasting insulin was significantly lower in male syndecan-3-1- mice than in male wild-type mice. Perhaps due to the small sample size, this effect was not statistically significant $(P=0.16)$ for the female syndecan-3-/- mice. Finally, glucose tolerance was not significantly different when mice were raised on a diet low in fat (15\% fat; rodent chow) (data not shown).

\section{Discussion}

Our data have demonstrated that syndecan- $3^{-/-}$mice are lean and resistant to diet-induced obesity. Additionally, our findings have shown that both male and female syndecan-3-1- mice (and female syndecan $-3^{+/-}$mice), when fed the HF diet, did not gain as diture compared with that of their wild-type controls, female syndecan-3 $3^{-/-}$mice exhibited even more pronounced elevations in energy expenditure than their female controls (Figure 6). For example, during the light cycle, female syndecan-3-/- mice burned approximately $15 \%$ more $\mathrm{kcal} / \mathrm{h}$ than wild-type mice burned, while male syndecan-3-/- mice utilized only $8 \%$ more $\mathrm{kcal} / \mathrm{h}$ than their wild-type control counterparts used.

Increased accumulation of body fat leads to insulin resistance and glucose intolerance. syndecan-3-1- mice partially resisted the obesity-inducing effects of the HF diet and showed improved glucose tolerance compared with the glucose tolerance of wild-type mice (Figure 7). Insulin concentrations during the IPGTT showed that syndecan- $3^{-/-}$mice were probably more insulin sensitive than wild-type mice were. In support of this finding were the lower
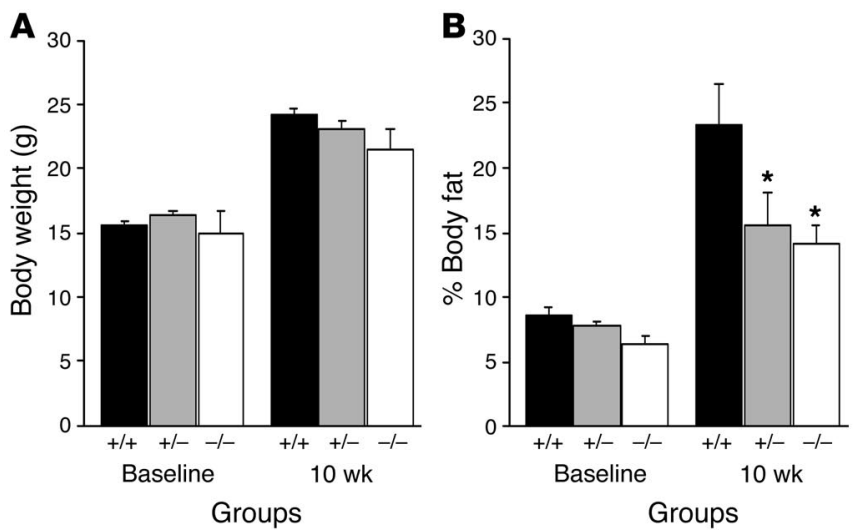

Figure 3

Body weight and percent body fat of female mice following a HF diet. (A and B) Body weight (A) and percent body fat (B) of wild-type $(n=3)$, syndecan $-3^{+-}(n=11)$, and syndecan- $3^{-/-}(n=4)$ female mice before and after 10 weeks of access to the HF diet ( ${ }^{*} P<0.05$ compared with wild-type mice, repeated-measures ANOVA). 

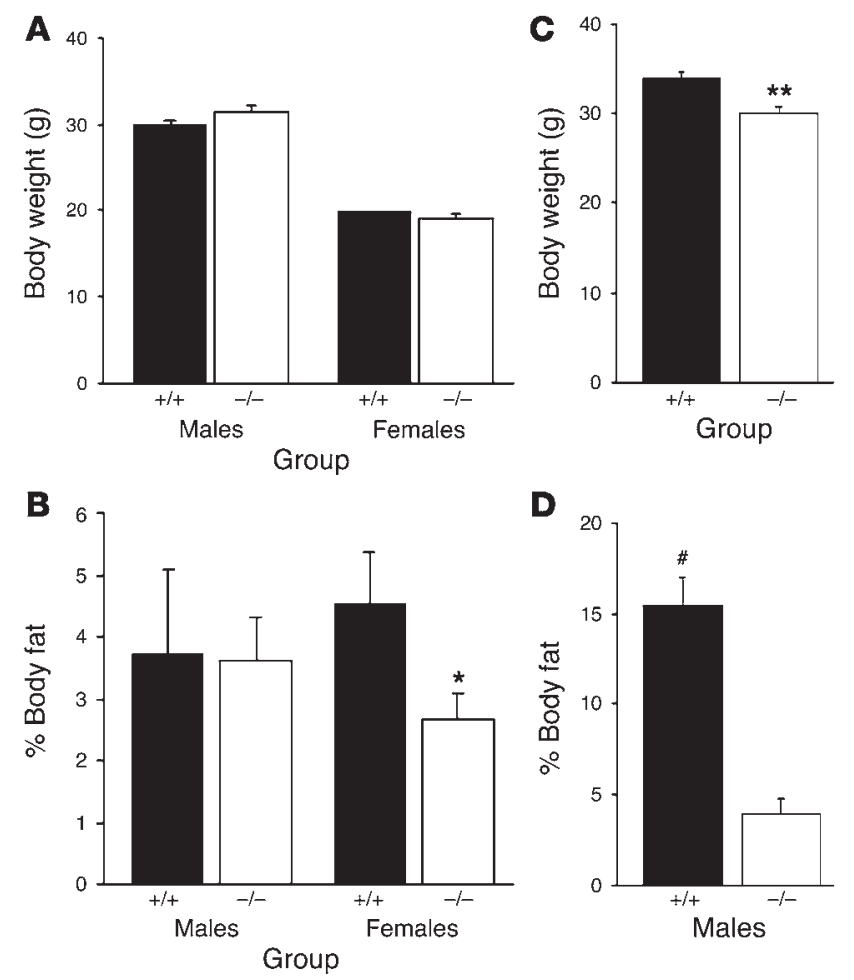

fasting insulin concentrations seen in male syndecan-3-/- mice than in wild-type mice (Figure 8). In contrast, regular chow-fed syndecan-3-1- and wild-type mice showed similar glucose tolerance (data not shown). Thus, syndecan $-3^{-/-}$mice are protected from the increased body fat that results from exposure to diets high in fat and they are also protected from the perturbations in normal glucose homeostasis also associated with such diets. Because lowered body fat would be predicted to improve glucose tolerance, these data cannot be used to determine if there is a role for syndecan-3 in glucose homeostasis independent of the role of syndecan-3 in body fat regulation.

These data make three important points about the function of syndecan-3. First, syndecan-3 deficiency results in a leaner body phenotype. Second, the role of syndecan-3 in body weight regulation is nutritionally sensitive. While syndecan-3-1- raised on chow exhibit reduced body fat compared with that of wild-type mice, the phenotype of syndecan-3-- mice is most apparent on diets high in fat, suggesting that there is a critical gene-environment interaction. Identifying such interactions is an important step in understanding the etiology of obesity that also clearly depends on gene-environment interactions $(8,10)$. Third, sex influences the expression of the syndecan-3-deficient phenotype. Male syndecan-3-deficient mice are partially protected from HF diet-induced obesity due to both reduced caloric intake and increased energy expenditure; in contrast, female syndecan-3-null mice resist diet-induced obesity mainly due to an increase in energy expenditure. Thus, syndecan-3 plays an important role in the regulation of energy balance in both male and female mice, although the mechanism by which it is manifested is sexually dimorphic.

A final critical question is about the relationship between syndecan-3 and other CNS systems involved in energy balance regulation. Several lines of evidence support the hypothesis that syndecan-3 serves to regulate CNS melanocortin signaling

\section{Figure 4}

Comparison of body weight and percent fat for syndecan-3-1- and wildtype mice. (A-D) Body weight ( $\mathbf{A}$ and $\mathbf{C}$ ) and percent body fat (B and D) of chow-raised (15\% fat) wild-type and syndecan-3-/- mice. Young (12-week-old) mice (male: $+/+, n=3$, and $-/-, n=3$; female: $+/+, n=7$, and $-/-, n=8$ ) and old (28-week-old) mice (male only; $+/+, n=10$, and $-/-, n=10)$ were compared $\left({ }^{\star} P<0.05\right.$ and ${ }^{\star \star} P<0.01$ compared with wild-type mice; ${ }^{P} P<0.01$ compared with syndecan $-3^{-1-}$ mice).

by acting as a coreceptor for the endogenous melanocortin-3/4 (MC3/4) receptor antagonist Agouti-related protein (AgRP). First, syndecan-3 is found in the CNS in regions of dense MC4 receptor expression (3). The distributions of both syndecan-3 and MC4 receptors overlap in many hypothalamic nuclei including the paraventricular nucleus, periventricular region, lateral hypothalamus, dorsomedial nucleus, and arcuate nucleus (11). Second, syndecan-3-/- mice are more sensitive to the food intake-reducing properties of the synthetic MC3/4 receptor agonist MTII (12). Third, actions of the CNS melanocortin system have been linked to food intake, energy expenditure, and glucose homeostasis, which we found to be altered in syndecan-3-/- mice. Fourth, overexpression or pharmacological administration of MC4 receptor antagonists results in increased consumption of HF diets (13, 14). Examining the orexigenic actions of full-length AgRP in syndecan-3-1- mice would be an obvious way to test this interaction in vivo. However, full-length AgRP is difficult to synthesize and is not available in sufficient quantities for such an experiment. In addition, syndecan-3 does not bind to the C-terminal domain of AgRP (AAs 83-132), so the use of the commercially available fragment would not test this hypothesis.

Despite the large pharmacological effect of exogenously administered AgRP fragments (14), mice that lack AgRP (15) develop diet-induced obesity in a manner similar to that of normal mice when given access to a HF diet (15). Thus, it would appear that AgRP is not necessary for the development of HF diet-induced obesity, and this questions whether the protective effects of syndecan-3 rely solely on its interaction with AgRP. Given the nature of these HSPGs, it is possible that syndecan-3 physically interacts with other molecules involved in the regulation
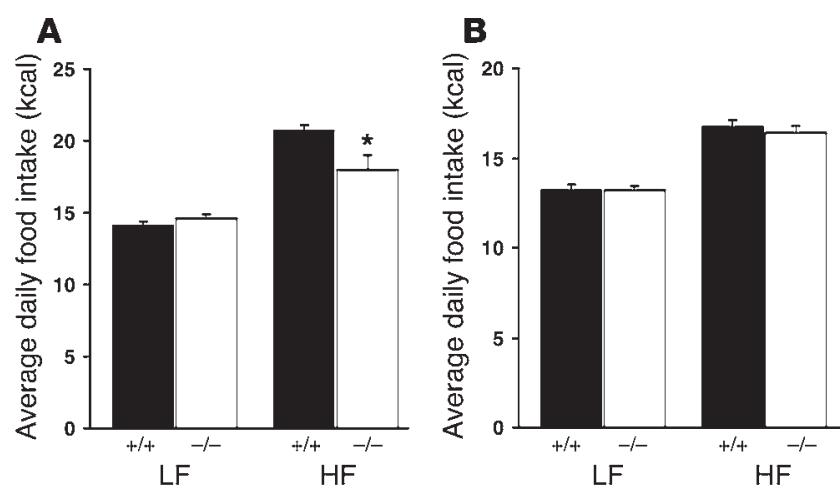

Figure 5

Food intake for syndecan-3--/ and wild-type mice. (A and B) Average daily food intake (kcal) for male (A) and female (B) mice on LF and $\mathrm{HF}$ diets. Wild-type and syndecan-3-1- mice were given LF and HF diets, and daily food intake was measured for 21 days $(n=5-9$ for each group). The average daily intake during the 21 days is presented ( ${ }^{\star} P<0.05$ wild type vs. syndecan-3-/- for each diet, Student's $t$ test). 
A

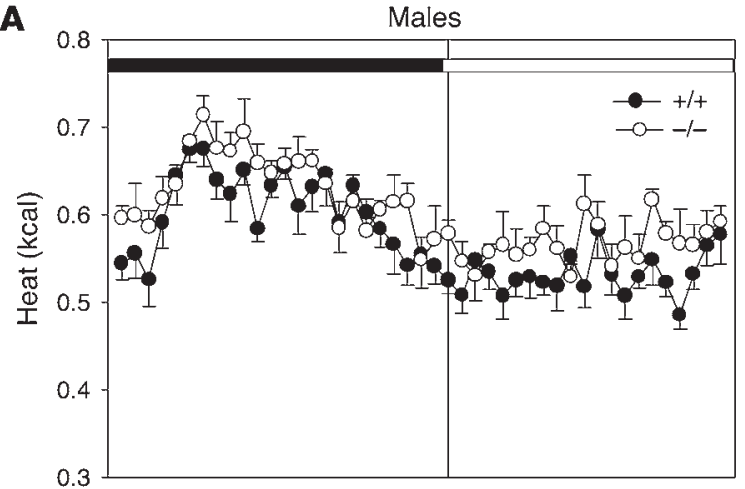

B

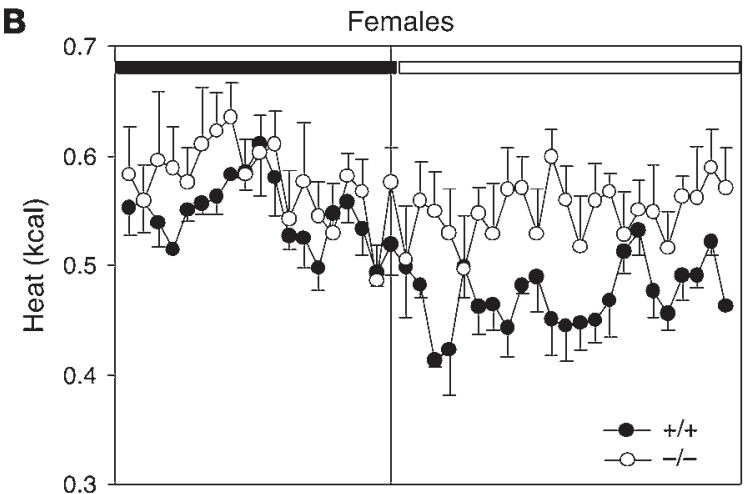

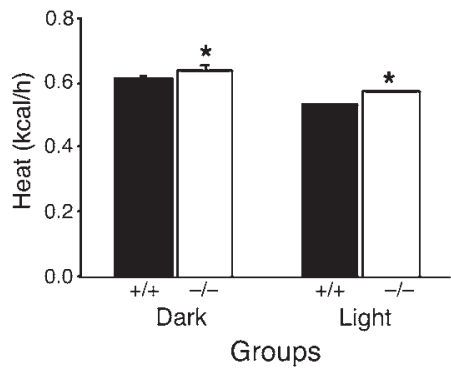

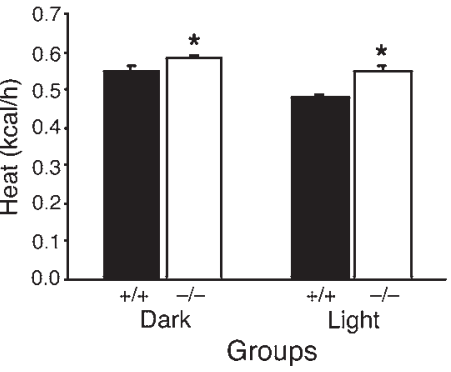

Figure 6

Comparison of oxygen consumption of syndecan-3-/- and wild-type mice. (A and B) Indirect calorimetry of male (A) and female (B) syndecan-3-1- mice $(n=4)$ and wild-type mice $(n=4)$ on the $\mathrm{HF}$ diet. Heat $(\mathrm{kcal} / \mathrm{h})$ was measured for 24 hours in an indirect calorimeter. The mean energy expenditure $(\mathrm{kcal} / \mathrm{h})$ was calculated separately during the light and dark cycles for each group of mice. Syndecan-3-1- mice exhibited greater energy expenditure during both the light and dark cycles $\left({ }^{*} P<0.001\right.$ wild type vs. syndecan-3 $3^{-/-}$for each sex, Student's $t$ test). of energy balance. Alternatively, it is possible that neuropeptide systems that regulate food intake are redundant enough to compensate for the complete absence of orexigenic signals such as AgRP or neuropeptide $Y$ but are less capable of compensating for the removal of modulators (coreceptors) and receptors such as syndecan-3 or MC4 receptors. Further experiments will be necessary to determine to what degree the phenotype of syndecan-3 deficiency depends on altered AgRP action.

Taken together, these data reveal an important role for syndecan-3 in the regulation of body weight. More importantly, if syndecan-3 does act to facilitate the action of AgRP on MC4 receptors, pharmacological inhibition of syndecan-3 may be a useful strategy to increase melanocortin signaling (receptor agonism) and to thereby treat obesity and its associated disruption of glucose homeostasis. This may be particularly useful, as selective MC4 receptor agonists have been associated with sexual side effects associated with extrahypothalamic MC4 receptors (16). Because syndecan-3 has a more narrow distribution, it may provide a more selective means to achieve increased melanocortin signaling and provide a novel therapeutic option for the treatment of obesity.

\section{Methods}

\section{Mouse strains}

Mice were maintained on a 12-hour/12-hour light/dark cycle with food and water provided ad libitum unless otherwise specified in the experimental design. Both strains of mice, syndecan-3-/- mice and wild-type C57BL/6J mice (derived from mice originally obtained from Jackson Laboratories), were bred and raised at the animal facility at the University of Cincinnati (Cincinnati, Ohio, USA) and all procedures were approved by the Institutional Animal Care and Use Committee. The syndecan-3-1- mice used in the following experiments were offspring from crosses between syndecan $-3^{-/-}$mice that were previously backcrossed to C57BL/6J mice for more than eight generations. The generation of the syndecan-3-/- mice was described in the original report by Reizes et al. (3). For the majority of the studies, mice were generated through breeding of homozygous mice. An additional group of mice were generated from breeding mice that were heterozygous for syndecan-3 deficiency to yield syndecan-3 $3^{+/+}$, syndecan $-3^{+/-}$, and syndecan $-3^{-/-}$littermates. Female offspring were selected and given access to the HF diet (as described below) for 10 weeks.

\section{Feeding studies}

For all behavioral studies, mice were given ad libitum access to either the LF diet ( $11 \%$ fat by calories, $3.6 \mathrm{kcal} / \mathrm{g}$ ), the HF diet ( $41 \%$ fat by calories, $4.4 \mathrm{kcal} / \mathrm{g}$ ), or the chow diet (rodent chow, $15 \%$ fat by calories, $3.4 \mathrm{kcal} / \mathrm{g}$; Harlan-Teklad). The LF and HF diets were purchased from Dyets Inc. and were butter based. For the long-term feeding study (14 weeks), male and female syndecan-3-/- and wild-type mice ( $n=6-10$ per group) were weaned at 28 days of age and were begun on the LF or HF diet on day 35 of age. An additional group of mice was begun on the HF diet only for 10 weeks. The mice used for this study were syndecan-3-/- $(n=4)$, syndecan-3 $3^{+-}(n=11)$, or wild type $(n=3)$. Mice were housed together by group and were weighed weekly. Individual daily food intake was not measured during the longterm studies (14 and 10 weeks on HF diet), as the mice were not housed individually. During a separate short-term study (3 weeks), 16- to 20-weekold weight-matched mice (syndecan-3-/- and wild type; $n=5-9$ per group) were individually housed and food intake was measured. Each mouse was provided with either the LF or HF diet. Fresh HF and LF diets were given twice per week. In a separate study, body weights and body compositions of young (12-13 weeks of age) and old (28 weeks of age) syndecan-3-/- and wild-type mice raised on chow were also compared. 

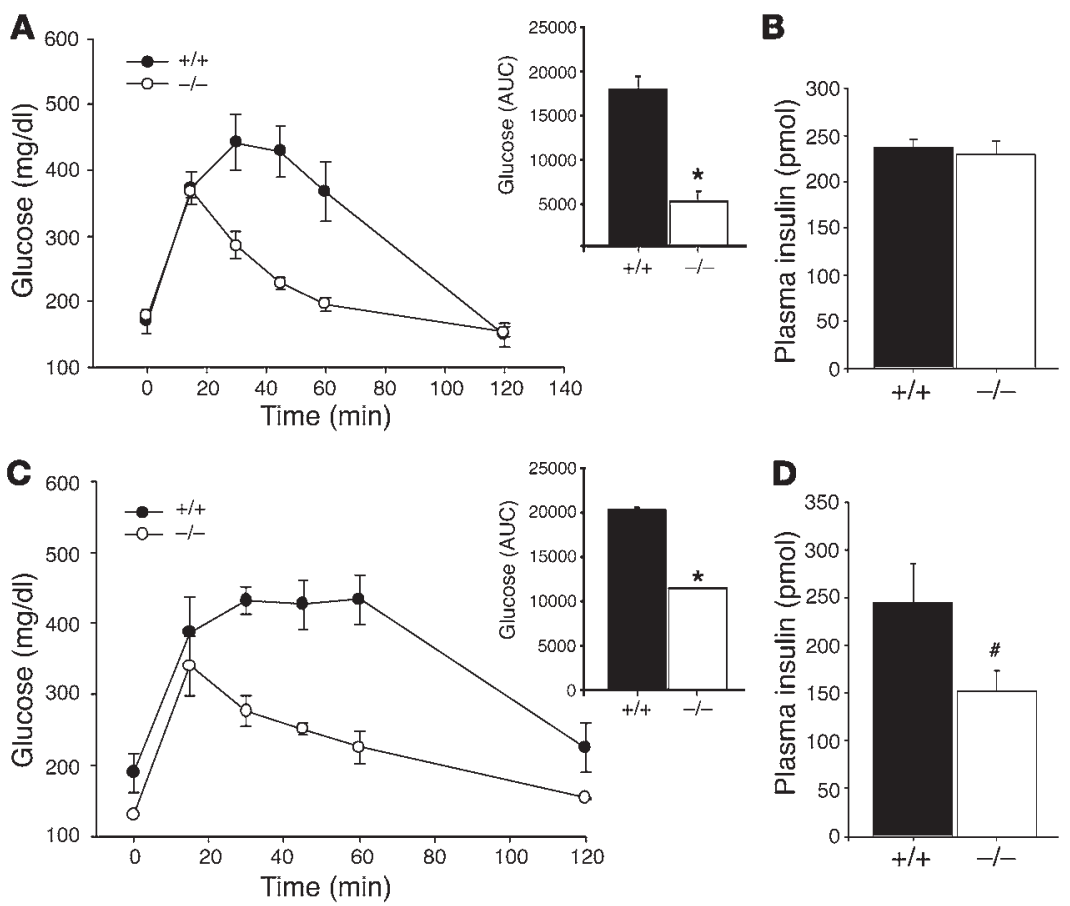

\section{Figure 7}

Glucose tolerance of syndecan-3-1- and wild-type mice. (A and $\mathbf{C}$ ) Glucose tolerance of female $(\mathbf{A})$ and male $(\mathbf{C})$ syndecan-3-1- mice $(n=3)$ compared with wild-type mice ( $n=3)$. Glucose ( $20 \%$ D-glucose) was administered intraperitoneally and tail blood samples were drawn for glucose measurements (at 0, 15, 30, 45,60 , and 120 minutes after glucose administration). The area under the curve (AUC) was calculated (using trapezoidal analysis) during the IPGTT and was compared between wild-type and syndecan-3-/mice using a Student's $t$ test $\left({ }^{*} P<0.001\right)$. (B and D) Plasma insulin was measured in female (B) and male (D) syndecan-3-/- and wild-type mice. In a second IPGTT administered identically to the first, a blood sample was drawn 15 minutes following glucose administration for analysis of plasma insulin. Insulin was compared between wild-type and syndecan-3-1mice using a Student's $t$ test $(\# P<0.05)$. No difference was seen between plasma insulin levels between female mice.

\section{Glucose tolerance tests}

At the end of the long-term feeding study (14 weeks on the HF diet), mice on the HF diet were administered an IPGTT $(1.5 \mathrm{~g} / \mathrm{kg}$ of $20 \% \mathrm{D}$-glucose, injected intraperitoneally). Three age-matched male and female mice from each genotype raised on the HF diet were used in the experiments. Additionally, a second cohort of adult age- and weight-matched wild-type and syndecan $-3^{-/-}$mice raised on chow were given an IPGTT ( $n=4$ per group). For the glucose tolerance tests, mice were made to fast overnight (16-19 hours). Baseline and test samples were all obtained from the tail vein. Blood glucose was measured at $0,15,30,34,60$, and 120 minutes after glucose administration with the One Touch glucometer (LifeScan). For plasma insulin measurements, a second IPGTT test was administered under the same experimental conditions a week later and one blood sample was taken from the tail vein 10-15 minutes after the glucose injection. Plasma insulin was measured by RIA as described previously (17).

\section{Indirect calorimetry}

Indirect calorimetry was performed using the Columbus Instruments Oxymax 5.41 system. Adult age-matched syndecan-3-/- $(n=4$ for each sex $)$ and wild-type ( $n=4$ for each sex) mice were placed in individual metabolic chambers 2 hours prior to the onset of the dark cycle. Mice remained in the chambers for 24 hours with ad libitum access to HF diet and water. Samples were collected approximately every 30 minutes. For each time point, the samples for each group were averaged. Average $\mathrm{kcal} / \mathrm{h}$ values were compared during the light and dark cycles for both groups. The flow rate to the individual chambers was set at 0.6 liters per minute, with room air as the reference. The system measuring time was set for 60 seconds and represents the amount of time during which the indirect calorimeter monitors the gas concentrations. The indirect calorimeter takes as many readings as possible during this interval and derives a mean value. The system settling time was set at $120 \mathrm{sec}-$ onds and represents the amount of time between the opening of the intake valve and the onset of the measuring time. This time allows for the complete purging of any residual gas in the system. Prior to the indirect calorimetry experiments, mice were maintained on the HF diet for 4 weeks.

\section{Body composition analysis}

Method 1: Lyophilization and ether extraction. For the long-term diet study (14 weeks on the HF diet), body composition was analyzed by lyophilization and ether extraction. Three male and female syndecan-3-1- and wild-type mice from the long-term diet study were sacrificed 1 week after the final IPGTT. Carcasses were saved and stored at $-80^{\circ} \mathrm{C}$. Mouse carcasses were freeze-dried for 3 days with a lyophilizer (Labconco) for the withdrawal of total body water content. After lyophilization, the carcasses were placed in a large soxlet and were flushed with boiling ether for 8 hours. The carcasses were then dried and weighed, and body water, body fat, and lean tissue mass were determined as percentage of the animal's final body weight.

Method 2: NMR. For the chow-raised mice and the female mice in the 10-week HF diet study that included all three genotypes (wild type, syndecan-3 $3^{+-}$, and syndecan-3---), body composition was analyzed with a custom-designed rodent quantitative NMR apparatus (Echo MRI Whole
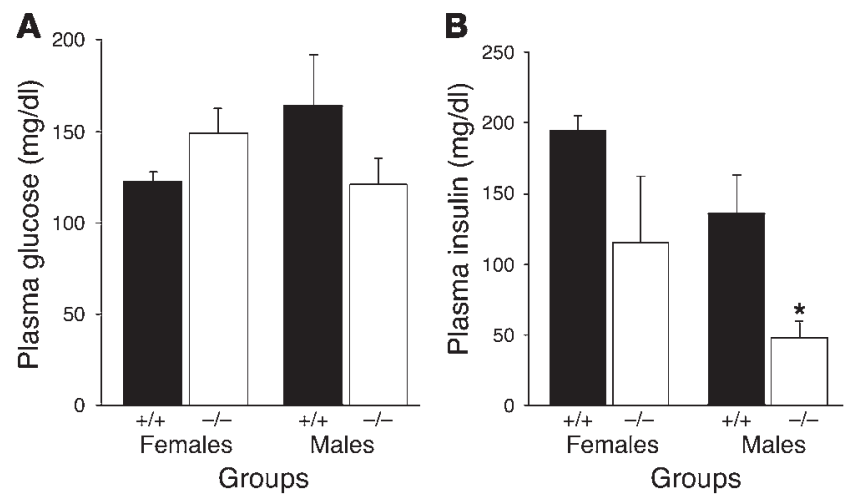

Figure 8

Fasting plasma glucose and insulin following a HF diet. (A and B) Fasting plasma glucose $(\mathbf{A})$ and plasma insulin $(\mathbf{B})$ of wild-type $(n=3)$ and syndecan- $3^{-/-}(n=3)$ mice after 14 weeks on the HF diet $\left({ }^{*} P<0.05\right.$, Student's $t$ test). 
Body Composition Analyzer; Echo Medical Systems). This allowed us to compare changes in body composition in the same animals over time. The method is described in ref. 18. Briefly, each mouse was transported into a room that housed the NMR and placed (without anesthesia) in a Plexiglas tube that was then inserted into the NMR for body composition analysis. Body fat and lean tissue were measured during the test, which takes less than 60 seconds to perform.

\section{Statistical analysis}

Data were analyzed using parametric statistics (Sigma Stat version 2.03). Repeated-measures two-way ANOVA was utilized for analysis of body weights and body composition during the long-term diet studies (14 and 10 weeks on the HF diet). For comparisons of body composition measures, food intake (kcal), mean $\mathrm{kcal} / \mathrm{h}$, glucose area under the curve, fasting plasma glucose, and insulin, a Student's $t$ test was utilized. $P$ values less than 0.05 were considered statistically significant.

1. Spiegelman, B.M., and Flier, J.S. 2001. Obesity and the regulation of energy balance. Cell. 104:531-543.

2. Bernfield, M., et al. 1999. Functions of cell surface heparan sulfate proteoglycans. Annu. Rev. Biochem. 68:729-777.

3. Reizes, O., et al. 2001. Transgenic expression of syndecan-1 uncovers a physiological control of feeding behavior by syndecan-3. Cell. 106:105-116.

4. Williams, G., et al. 2001. The hypothalamus and the control of energy homeostasis: different circuits, different purposes. Physiol. Behav. 74:683-701.

5. Carey, D.J., et al. 1992. Molecular cloning and characterization of $\mathrm{N}$-syndecan, a novel transmembrane heparan sulfate proteoglycan. J. Cell Biol. 117:191-201.

6. Berndt, C., Casaroli-Marano, R.P., Vilaro, S., and Reina, M. 2001. Cloning and characterization of human syndecan-3. J. Cell. Biochem. 82:246-259.

7. Woods, S.C., Seeley, R.J., Rushing, P.A., D'Alessio, D.A., and Tso, P. 2003. A controlled high-fat diet induces an obese syndrome in rats. J. Nutr.

\section{Acknowledgments}

The authors thank Jennifer Schurdak, Kimberly Madaris, Moctar Karimou, and Kay Ellis for their technical expertise. This work was supported by grants from the NIH (DK065434, DK054080, DK056863, DK064885, and DK17844) and funds from the Procter \& Gamble Company.

Received for publication November 24, 2003, and accepted in revised form August 31, 2004.

Address correspondence to: April D. Strader, Genome Research Institute, University of Cincinnati, 2170 East Galbraith Road, Building 43 ML 0506, Reading, Ohio 45237, USA. Phone: (513) 558-4703; Fax: (513) 558-8990; E-mail: April.Strader@uc.edu.

April D. Strader and Ofer Reizes contributed equally to this work.

133:1081-1087.

8. West, D.B., and York, B. 1998. Dietary fat, genetic predisposition, and obesity: Lessons from animal models. Am. J. Clin. Nutr. 67:505S-512S.

9. Swinburn, B.A., Caterson, I., Seidell, J.C., and James, W.P. 2004. Diet, nutrition and the prevention of excess weight gain and obesity. Public Health Nutr. 7:123-146.

10. Leibel, R.L., Chung, W.K., and Chua, S.C., Jr. 1997. The molecular genetics of rodent single gene obesities. J. Biol. Chem. 272:31937-31940.

11. Liu, H., et al. 2003. Transgenic mice expressing green fluorescent protein under the control of the melanocortin-4 receptor promoter. J. Neurosci. 23:7143-7154.

12. Reizes, O., et al. 2003. Syndecan-3 modulates food intake by interacting with the melanocortin/AgRP pathway. Ann. N. Y. Acad. Sci. 994:66-73.

13. Koegler, F., Schaffhauser, R., Mynatt, R., York, D., and Bray, G. 1999. Macronutrient diet intake of the lethal yellow agouti (Ay/a) mouse. Physiol. Behav.
67:809-812.

14. Hagan, M.M., et al. 2000. Long-term orexigenic effects of AgRP-(83---132) involve mechanisms other than melanocortin receptor blockade. Am. J. Physiol. Regul. Integr. Comp. Physiol. 279:R47-R52.

15. Qian, S., et al. 2002. Neither agouti-related protein nor neuropeptide $\mathrm{Y}$ is critically required for the regulation of energy homeostasis in mice. Mol. Cell. Biol. 22:5027-5035.

16. Van der Ploeg, L.H., et al. 2002. A role for the melanocortin 4 receptor in sexual function. Proc. Natl. Acad. Sci. U. S. A. 99:11381-11386.

17. Ensinck, J.W., Laschansky, E.C., Vogel, R.E., and D'Alessio, D.A. 1991. Effect of somatostatin-28 on dynamics of insulin secretion in perfused rat pancreas. Diabetes. 40:1163-1169.

18. Taicher, G.Z., Tinsley, F.C., Reiderman, A., and Heiman, M.L. 2003. Quantitative magnetic resonance (QMR) method for bone and wholebody-composition analysis. Anal. Bioanal. Chem. 377:990-1002. 